\title{
Acute Subdural Hematoma during Tack-up Suture of the Dura Mater -Case Report-
}

\author{
Nobuhiko AOKI
}

Department of Neurosurgery, Tokyo Metropolitan Fuchu Hospital, Fuchu, Tokyo

\begin{abstract}
A rare neurosurgical event is described, in which an acute subdural hematoma developed during tack-up suture of the dura mater after evacuation of an acute epidural hematoma. The source of the second hematoma was determined to have been a ruptured bridging vein. The author discusses the potential risk of tack-up suture when there is a large gap between the dura mater and the inner surface of the skull.
\end{abstract}

Key words: acute epidural hematoma, acute subdural hematoma, intracranial bridging veins, neurosurgical complications

\section{Introduction}

It is well known that acute subdural hematoma in the absence of cerebral contusion may be caused by rupture of bridging veins. ${ }^{1,2)}$ This lesion is exclusively traumatic in origin; however, a unique case is reported here, in which a bridging vein ruptured during tack-up suture of the dura mater after evacuation of an acute epidural hematoma. Although this would not seen to be an extremely rare event, to the author's knowledge no such case has been reported previously.

\section{Case Report}

An 11-year-old boy was brought to the Department of Neurosurgery, Tokyo Metropolitan Fuchu Hospital on May 14, 1986, in a comatose state. He had a history of psychomotor retardation and epilepsy, for which he had been treated on an outpatient basis. His family reported that he had complained of a headache at 8:00 p.m. on the previous day. At that time, although he was not definitely known to have suffered head trauma, his scalp was reportedly swollen in the left parietal region. Early in the morning of the following day, he began vomiting frequently, and then he was brought to this hospital by ambu-

Received March 11, 1987; Accepted March 2, 1988 lance in coma.

On admission, the physical findings were unremarkable except for a subgaleal hematoma in the left parieto-occipital region. He was comatose on neurological examination. The left pupil was larger than the right, and right hemiplegia was noted. No fractures were demonstrated by skull x-rays. A computed tomographic (CT) scan (Fig. 1) revealed a massive acute epidural hematoma on the left side, with ventricular distortion. There was no associated cerebral contusion or subdural hematoma. Results of laboratory tests, including coagulation studies, were normal.

He underwent an emergency left parietal craniotomy in the supine position with his head fully turned to the right. Although no fracture was seen, a contusion in the subcutaneous tissue confirmed that the lesion was due to head trauma. Approximately 80 gm of epidural clots were removed, but the source of the epidural hematoma was not identified. After evacuation of the clots, the dura mater was suspended a considerable distance from the inner surface of the skull. Tack-up suture of the dura at the margins of the craniotomy was therefore begun. During this procedure, it was noticed that the dura rapidly tightened. Immediate CT scanning was considered in the interest of clarifying the cause of the rapid progression of intracranial hypertension, but it was decided that the ipsilateral subdural space should be 


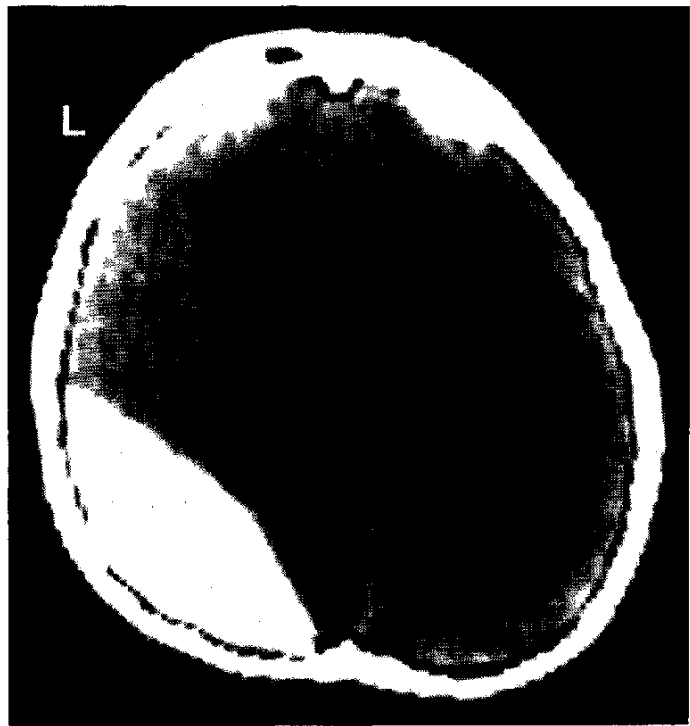

Fig. 1 CT scan on admission demonstrating a massive acute epidural hematoma in the left parietooccipital region.

explored prior to CT examination. A dural incision disclosed a fresh, homogeneously clotted hematoma about $3 \mathrm{~cm}$ in thickness, covering the entire left cerebral hemisphere.

Evacuation of the subdural hematoma revealed the source of bleeding to be a ruptured bridging vein draining into the superior sagittal sinus. No cerebral contusion was observed, and the ruptured vein was easily cauterized. He fully recovered within 1 week of surgery.

\section{Discussion}

Following the removal of an intracranial mass lesion of significant size, the dura mater commonly suspends from the skull. In this situation, tack-up suture of the dura is usually performed to prevent the development of postoperative epidural hematoma. This procedure eventually could cause tearing of bridging or cortical veins adherent to the inner surface of the dura, when there is a large gap between the dura and the skull. The author suppose that this intra- or postoperative complication might have been encountered at most neurosurgical institutes; however, to the author's knowledge, no such case has been reported to date.
Evacuation of acute epidural hematoma has been reported, although rarely, to be followed by rapid development of intracranial hypertension., ${ }^{3,4)}$ The lesions responsible for its occurrence include ipsilateral or contralateral delayed intracerebral hematoma ${ }^{3)}$ and acute epidural hematoma on the contralateral side. ${ }^{4)}$ Both are thought to be secondary to rapid decompression. Rebleeding from bridging veins initially ruptured at the time of the head trauma is another possibility. However, it is reasonable to speculate that the suturing procedure contributed to the development of acute subdural hematoma through the rupture of bridging veins.

This case indicates that acute subdural hematoma is one cause of rapid elevation of intracranial pressure following removal of an acute epidural hematoma. In the event of acute intraoperative intracranial hemorrhage of unknown origin, dural incision and exploration may be advisable prior to CT scanning.

\section{Acknowledgments}

The author wishes to express his appreciation to Dr. Taeko Summerville, Department of Neurosurgery, Neurological Institute, Tokyo Women's Medical College, for her permission to present this case.

\section{References}

1) Aoki $\mathrm{N}$, Masuzawa $\mathrm{H}$ : Infantile acute subdural hematoma. Clinical analysis of 26 cases. $J$ Neurosurg 61: 273-280, 1984

2) Bakay L, Glasauer FE: Head Injury. Boston, Little Brown and Company, 1980, pp 199-200

3) Taneda $M$, Irino $T$ : Enlargement of intracerebral haematomas following surgical removal of epidural haematomas. Acta Neurochir (Wien) 51: 73-82, 1979

4) Tani S, Yamanouchi Y, Kawamura Y, Matsumura H, Nogawa T: Acute contralateral epidural hematoma developing during decompressive surgery for traumatic intracranial hematoma. Report of three cases. Neurol Med Chir (Tokyo) 23: 152-156, 1983 (in Japanese)

Address reprint requests to: N. Aoki, M.D., Department of Neurosurgery, Tokyo Metropolitan Fuchu Hospital, 2-9-2 Musashidai, Fuchu, Tokyo 183, Japan. 